\title{
Jaceosidin Induces Apoptosis in Human Ovary Cancer Cells through Mitochondrial Pathway
}

\author{
Wen Lv, ${ }^{1}$ Xia Sheng, ${ }^{2}$ Ting Chen, ${ }^{2}$ Qiang $X u_{,}{ }^{2}$ and Xing Xie ${ }^{1}$ \\ ${ }^{1}$ The Second Affiliated Hospital, Women's Hospital, School of Medicine, Zhejiang University, Hangzhou 310009, China \\ ${ }^{2}$ State Key Laboratory of Pharmaceutical Biotechnology, School of Life Sciences, Nanjing University, Nanjing 210093, China
}

Correspondence should be addressed to Xing Xie, xiex@zju.edu.cn

Received 14 January 2008; Accepted 6 July 2008

Recommended by Wolfgang Schulz

We examined the antiproliferation effect of Jaceosidin (4', 5, 7-trihydroxy-3', 6-dimethoxyflavone) isolated from the herb of Artemisia vestita Wall on several human cancer cell lines. Jaceosidin significantly reduced the proliferation of CAOV-3, SKOV3, HeLa, and PC3 cells in a concentration-dependent manner. A time-dependent inhibition was also observed in CAOV-3 cells by Jaceosidin. By flow cytometric analysis, we found that Jaceosidin treatment resulted in an increased apoptosis in CAOV-3 cells. The cells treated with Jaceosidin exhibited a decreased mitochondrial membrane potential. Jaceosidin also increased the level of cleaved caspase- 9 and induced the cleavage of caspase- 3 and poly (ADP-ribose) polymerase (PARP), while caspase- 3 inhibitor Z-DEVD-FMK significantly reversed the proapoptotic effect of Jaceosidin in CAOV-3 cells. Moreover, Jaceosidin elevated the level of cytochrome $c$ in cytosol. These findings suggest that the anticancer effect of Jaceosidin may be contributed by an induction of apoptosis involving cytochrome $c$ release from mitochondria to cytosol.

Copyright (C) 2008 Wen Lv et al. This is an open access article distributed under the Creative Commons Attribution License, which permits unrestricted use, distribution, and reproduction in any medium, provided the original work is properly cited.

\section{INTRODUCTION}

The normal cell function and tissue homeostasis are maintained by a balance between proliferation and apoptosis. Cancer is a typical disorder in which clones of malignant cells escape such balance and proliferate inappropriately without compensatory apoptosis [1]. Generally, the growth rate of preneoplastic or malignant cells outpaces that of normal cells as a consequence of malfunctioning or deregulation of their cell growth and cell death machineries [2]. The success of cancer therapies, therefore, greatly relies on the extent to which they preferentially induce tumour cell death while allowing survival of normal tissue. Blockade of proliferation or induction of apoptosis has been recognized as a rational approach to eliminate genetically damaged or preneoplastic cells before any malignancy manifests [3-6].

As an important resource of anticancer agents, many plant-derived substances have been shown to have various bioactivities. For example, some of the Artemisia plants widely used in traditional oriental medicine have been reported to show antimutagenic and anti-inflammatory effects [7-9]. Our previous study has also observed that the ethanol extract from Artemisia vestita Wall showed an inhibitory effect on endotoxin-induced sepsis through suppressing MAPKs and NF- $\kappa \mathrm{B}$ signaling in macrophages [10]. From the plant, we further found an anticancer principle, a flavone jaceosidin. So far, there are only a few reports on the pharmacological activities of this compound, such as inhibition of COX-2 and MMP-9 in human mammary epithelial cells [11], suppression of E6 and E7 oncoproteins of HPV 16 [12], and induction of apoptosis in ras-transformed human breast epithelial cells [13]. Based on the antiproliferation and apoptosis-inducing effects of jaceosidin, thus, the present study aims at demonstrating the mechanisms underlying the effects against cancer cells from the aspect of apoptosis pathway.

\section{MATERIALS AND METHODS}

\subsection{Cell lines}

Human ovary cancer cells CAOV-3, SKOV-3, PC3, and HeLa cells were obtained from ATCC. All the cells were maintained in DMEM (Life Technologies Inc., Grand Island, NY, USA) supplemented with 10\% FBS (Life Technologies Inc.), $100 \mathrm{U} / \mathrm{mL}$ penicillin, and $100 \mu \mathrm{g} / \mathrm{mL}$ streptomycin, and 
incubated at $37^{\circ} \mathrm{C}$ in a humidified atmosphere containing $5 \% \mathrm{CO}_{2}$ in the air.

\subsection{Drugs and reagents}

Jaceosidin, 4', 5, 7-trihydroxy-3', 6-dimethoxyflavone (see Figure 1(a)), was isolated from Artemisia vestita Wall, a traditional Tibetan medicine. The following reagents were purchased as follows. 3-(4, 5-dimethyl-2-thiazyl)-2, 5-diphenyl2H-tetrazolium bromide (MTT) (Sunshine Biotechnology, Nanjing, China); antibodies to poly (ADP-ribose) polymerase (PARP), caspase-3, caspase-9, cytochrome $c$ oxidase subunit IV (COX-IV), and cytochrome $c$ (Cell signaling Technology, Beverly, Mass, USA); antibody to tubulin (Stanta Cruz Biotechnology, Stanta Cruz, Calif, USA); peroxidaselabeled antirabbit antibody, peroxidase-labeled antimouse antibody (KPL, Gaithersburg, Md, USA); 5, 5', 6, 6' tetrachloro-1, 1', 3, 3'-tetraethyl-benzimidazolcarbocyanine iodide (JC-1) (Molecular Probes, Eugene, Ore, USA). ZDEVD-FMK (BD biosciences, San Jose, Calif, USA).

\subsection{MTT assay}

Five thousands of cells per well were cultured in a 96well-plate for first 24 hours. Then, they were incubated with different concentrations of jaceosidin $(10,20,40$, and $80 \mu \mathrm{M})$ for further 48 hours. For the time course assay, the incubation time with jaceosidin was 24, 48, and 72 hours, respectively. After incubation, MTT was dissolved in PBS at $5 \mathrm{mg} / \mathrm{mL}$ and added to culture media at a final concentration of $0.5 \mathrm{mg} / \mathrm{mL}$. After incubation at $37^{\circ} \mathrm{C}$ for 4 hours, the media were removed, $100 \mu \mathrm{L}$ DMSO were added to each well to dissolve purple crystals of formazan. The plate was shaken for 10 minutes to allow complete solubilization. Spectrophotometric absorbance at $540 \mathrm{~nm}$ was read on a 96well plate reader.

\subsection{Assay for cell apoptosis}

Jaceosidin or quercetin was added into 12-well-plate at indicated concentrations. In the inhibitory assay, $100 \mu \mathrm{L} \mathrm{Z}$ DEVD-FMK, a capase-3 inhibitor, was added to each well along with the compounds. Twenty four-hours later, the cells were collected, washed, and resuspended in PBS. Apoptotic cell death was identified by double supravital staining with recombinant FITC (fluorescein isothiocyanate)-conjugated Annexin V and PI, using the Annexin V-FITC Apoptosis Detection kit (Becton Dickinson, Frankly Lakes, NJ, USA) according to the manufacturer's instructions. Flow cytometric analysis was performed immediately after supravital staining. Data acquisition and analysis were performed in a Becton Dickinson FACSCalibur flow cytometer using CellQuest software.

\subsection{Assay for cell mitochondrial membrane potential}

The JC-1 dye assay was used for determination of reduction in mitochondrial membrane potential during apoptosis. Briefly, jaceosidin or quercetin was added to 12-well-plate

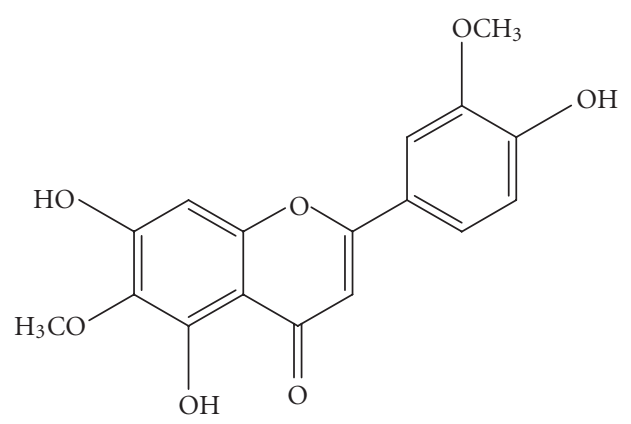

(a)

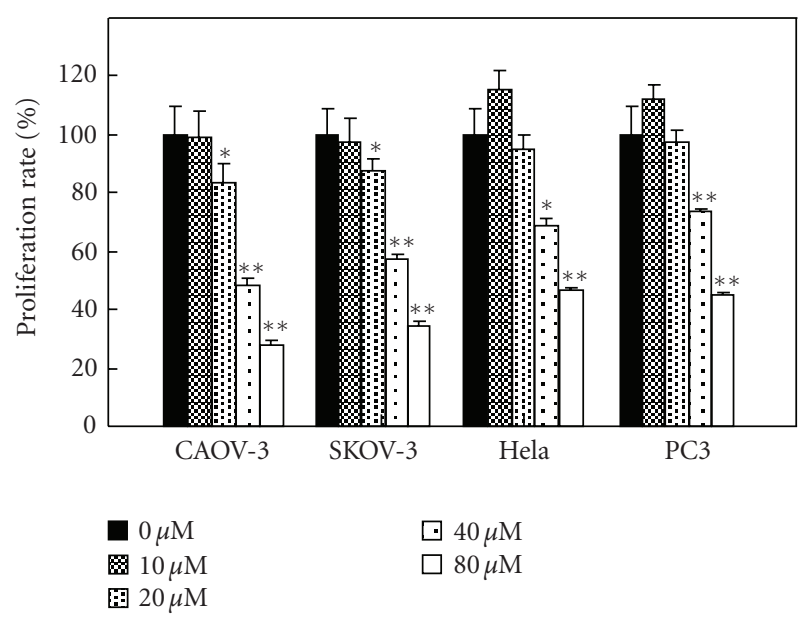

(b)

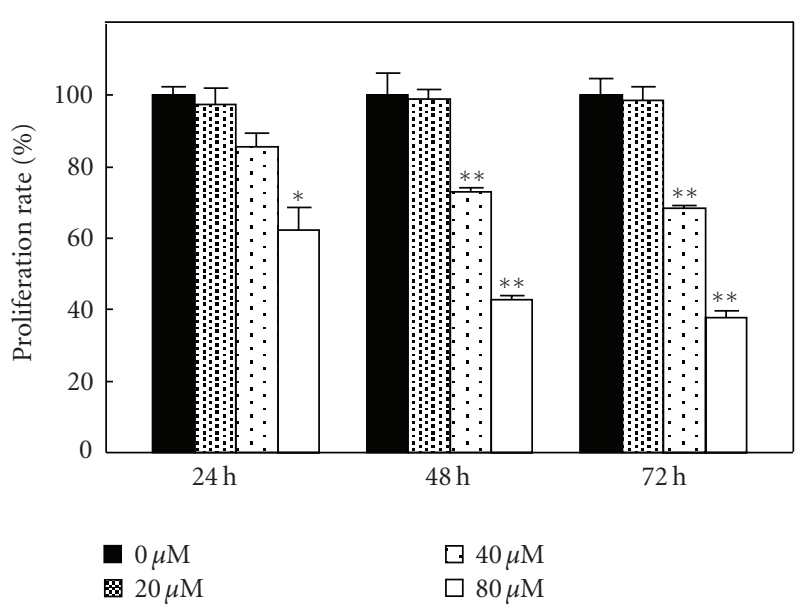

(c)

FIgURE 1: (a) Structure of jaceosidin. (b) Jaceosidin inhibits proliferation of CAOV-3, SKOV-3, PC3, and HeLa cells in a concentration-dependent manner. Cells were cultured in 96-wellplate for first 24 hours. Then, they were treated with different concentrations of jaceosidin for further 48 hours. (c) Jaceosidin inhibits proliferation in CAOV-3 cells in a time-dependent manner. The CAOV-3 cells were incubated with jaceosidin for 24, 48, and 72 hours, respectively. MTT assay was used to evaluate the proliferation rate. Each datum indicated mean \pm s.d. of three independent experiments. ${ }^{*} P<.05,{ }^{* *} P<.01$ versus control (Dunnett's test). 
at indicated concentration. Twenty-four hours later, cells were harvested and fixed in $0.5 \%$ PFA for 30 minutes. Then, cells were stained with $1 \mu \mathrm{L} \mathrm{JC-1}$ at $1 \mathrm{mg} / \mathrm{mL}$ in DMSO. After incubation with $\mathrm{JC}-1$ for 15 minutes at $37^{\circ} \mathrm{C}$, cells were analyzed for the decrease in red-orange fluorescence. Data acquisition and analysis were performed in a Becton Dickinson FACSCalibur flow cytometer using CellQuest software.

\subsection{Gel electrophoresis and western blot analysis}

Cells were incubated for 24 hours in the presence of indicated concentrations of jaceosidin or quercetin. After the incubation, the cells were harvested and lysed. Mitochondrial protein and cytosolic protein were isolated using Mitochondrial Fractionation Kit (Active Motif, Carlsbad, Calif, USA) according to the manufacturer's instructions. Proteins were quantified using a BCA protein assay kit (Pierce, Rockford, Ill, USA) according to the manufacturer's specifications. For western blot analysis, the proteins were electrophoresed on a 7.5 to $10 \%$ SDS-PAGE, followed by immunoblotting on PVDF membrane. Then, the membrane was blocked with $5 \%$ nonfat dry milk in TBS with $0.1 \%$ Tween 20 for 1 hour at room temperature. Finally, proteins were visualized using the enhanced chemiluminescence detection system (Cell Signaling Technology, Beverly, Mass, USA) after incubation with antibodies to PARP, caspase-3, caspase-9, cytochrome $c$, COX-IV, or tubulin overnight at $4^{\circ} \mathrm{C}$. Then, they were incubated with peroxidase-labeled antirabbit antibody or peroxidase-labeled antimouse antibody for 1 hour at room temperature. Peroxidase activity was visualized on X-ray film in darkroom.

\subsection{Statistical analysis}

Results were expressed as mean \pm s.d. of three independent experiments and each experiment included triplicate sets. Data were statistically evaluated by one-way ANOVA followed by Dunnett's test between control group and multiple dose groups, with the level of significance chosen as $P<.05$.

\section{RESULTS}

\section{Jaceosidin concentration-dependently and time-dependently inhibited proliferation of various cancer cells}

To examine the effects of jaceosidin on different kinds of tumour cells, exponentially growing cells were treated by jaceosidin for 48 hours. As shown in Figure 1(b), jaceosidin significantly reduced the proliferation of CAOV-3, SKOV3, PC3, and HeLa cells in a dose-dependent manner. The flavone also showed a time-dependent inhibition on the proliferation of CAOV-3 cells (see Figure 1(c)).

\section{Jaceosidin induced apoptosis in CAOV-3 cells in a caspase-3-dependent manner}

As shown in Figures 2(a) and 2(b), Annexin-V positive cells were considered as early and late apoptosis populations.
Treatment of jaceosidin induced apoptosis of CAOV-3 cells in a dose-dependent manner. Against the apoptosis induction, caspase-3 inhibitor Z-DEVD-FMK significantly reversed the proapoptotic effect of jaceosidin in CAOV-3 cells (see Figure 2(c)).

\section{Jaceosidin induced loss of mitochondrial membrane potential (MMP) and cytochrome c release from mitochondria to cytosol}

In Figure 3(a), mitochondrial potential alteration was examined upon jaceosidin treatment. CAOV-3 cells were incubated with jaceosidin or quercetin for 24 hours. As the result, a dose-dependent reduction in MMP was detected in the groups treated with jaceosidin. Quercetin also showed a strong decrease in MMP.

To confirm if jaceosidin induces apoptosis through mitochondrial pathway, we incubated CAOV-3 cells with jaceosidin for 24 hours. Then, we collected cells and isolated mitochondria protein. Thirty $\mu \mathrm{g}$ of each protein were subjected to Western blotting. Equal protein loading was confirmed by immunodetection of COX-IV for mitochondria protein or tubulin for cytosol protein. As shown in Figure 3(b), jaceosidin greatly increased the cytosol cytochrome $c$ and decreased the mitochondria cytochrome $c$ as compared with the control group.

\section{Jaceosidin activated caspase-3 and its related proteins to promote apoptosis}

To examine the status of the caspase- 3 protein, we performed Western blot analysis by using an anticaspase- 3 antibody, which recognizes both procaspase- 3 and cleaved caspase3 , the p17 large/active subunit of caspase-3. As shown in Figure 4, the p17 cleavage product appeared in lysates of CAOV-3 cells treated with $80 \mu \mathrm{M}$ jaceosidin and $10 \mu \mathrm{M}$ quercetin. Jaceosidin and quercetin also cleaved PARP to a band of $85 \mathrm{kDa}$. The amount of cleaved caspase- 9 was also increased significantly in the cells treated with quercetin, and slightly with jaceosidin.

\section{DISCUSSION}

In the present study, we first examined the effect of jaceosidin on some kinds of cancer cell lines (see Figure 1(b)). CAOV-3 cells were initiated from human ovary adenocarcinoma of a 54-years-old Caucasian female [14]. SKOV-3 is also a human ovary adenocarcinoma cell line, and it is resistant to tumor necrosis factor and to several cytotoxic drugs including diphtheria toxin, cis-platinum and adriamycin [15]. PC3, as an epithelial cell line, was established from a human grade IV prostatic adenocarcinoma metastatic to bone [16]. And HeLa cells are human cervix adenocarcinoma cells [17]. Though the inhibition of proliferation rate by jaceosidin was observed to different degrees against different kinds of cancer cells, a concentration-dependent action was shown in all 4 kinds of cell lines used. This finding suggests that jaceosidin may have a relatively wide spectrum of antitumor growth. In CAOV-3 cells, the compound also showed a 


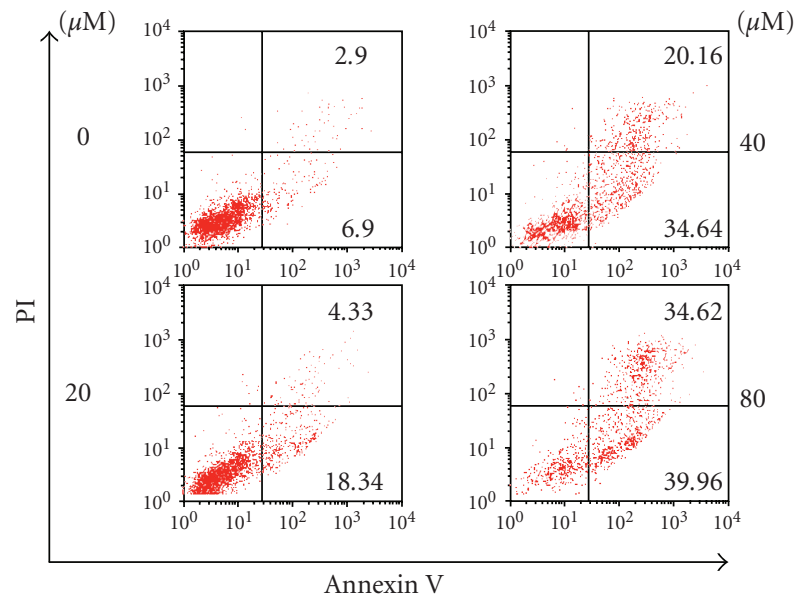

(a)

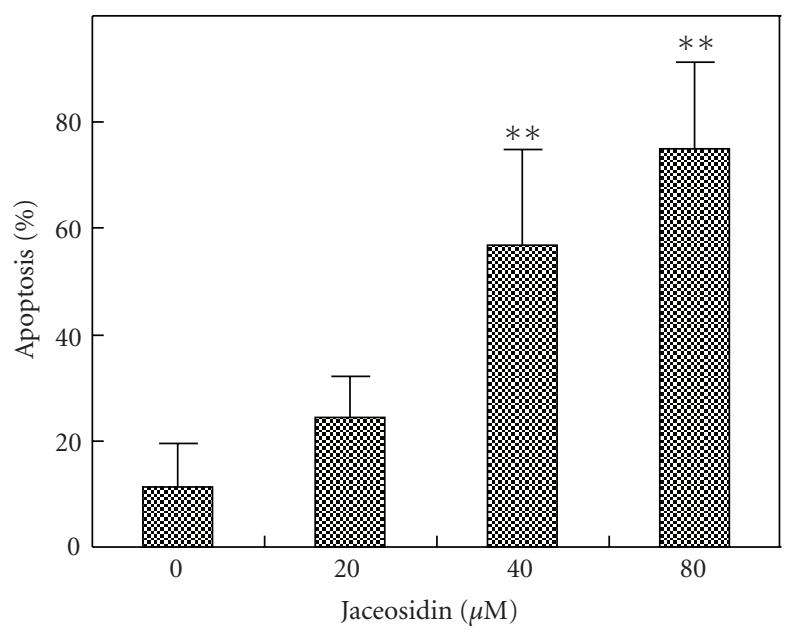

(b)

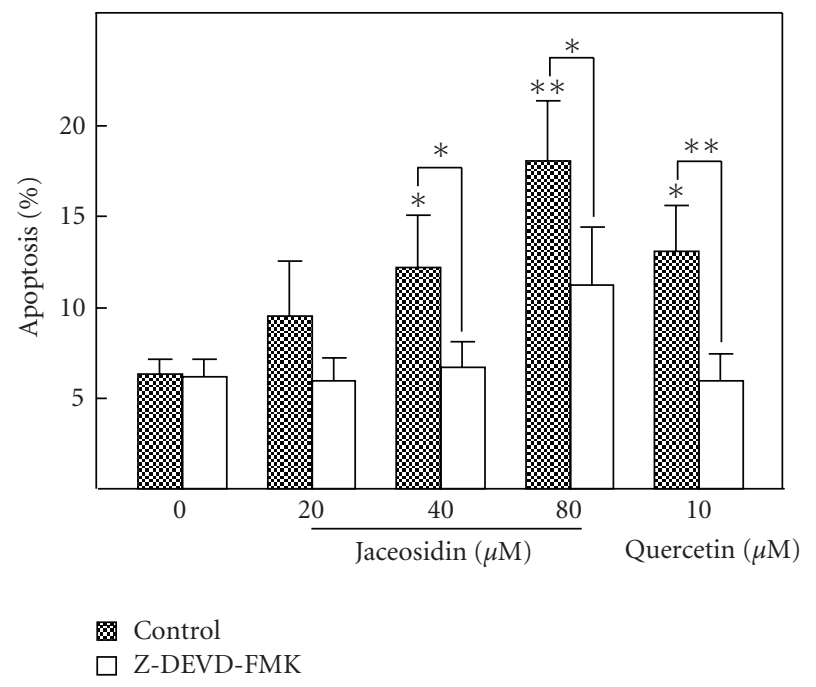

(c)

FIGURE 2: Jaceosidin promotes apoptosis of CAOV-3 cells in a caspase-3-dependent manner. CAOV-3 cells were seeded in 12-well-plate overnight, and then were treated with different concentrations of jaceosidin or $10 \mu \mathrm{M}$ quercetin in the presence or absence of $100 \mu \mathrm{M} Z$ DEVD-FMK for 24 hours. Annexin-V/PI stain was used to measure apoptosis rate. Apoptosis rate is measured by flow cytometry. (a) The results are representative of three separate experiments. (b) Data are expressed as a histogram of mean \pm s.d. of three independent experiments. (c) Effect of Z-DEVD-FMK on the proapoptotic activity of jaceosidin. Each datum indicated mean \pm s.d. of three independent experiments. ${ }^{*} P<.05, * * P<.01$ (Dunnett's test).

time-dependent inhibition against the cell proliferation (see Figure 1(c)). As the ovary cancer cell line CAOV-3 showed the most significant inhibitory rate, we chose it to do the following studies.

Apoptosis, a morphologically and biochemically defined form of cell death [18], plays a role in a wide variety of biologic systems. As apoptosis is often caused by chemotherapeutic drugs [19], we used annexin V-PI staining to study the apoptosis rate of cells treated with jaceosidin (see Figures 2(a) and 2(b)). The result indicated that jaceosidin could dose-dependently induce the apoptosis of CAOV-3 cells. The IC50 was less than $40 \mu \mathrm{M}$. Treatment of caspase-3 inhibitor led to a blockade of the proapoptotic effect of jaceosidin (see Figure 2(c)), indicating caspase-3 is involved in the underlying mechanism of jaceosidin's anticancer effect.
Chemotherapeutic agents have been described to induce apoptosis in two major routes: the extrinsic or death receptor-associated route and the intrinsic or mitochondrial route [20]. In the intrinsic route the execution phase is initiated by release of cytochrome $c$ and other polypeptides from the mitochondrial intermembrane space [21]. This release is accompanied by a dissipation of mitochondrial inner transmembrane potential [22]. It was reported that quercetin, another widely used flavone, has strong proapoptotic effect in cancer cells [23]. In the present study, we used it as positive control in the apoptosis assay for comparison. To further access the antitumour mechanism of jaceosidin, we observed that mitochondrial membrane potential decreased in a dose-dependent manner by jaceosidin (see Figure 3(a)). This result suggests that jaceosidin probably promotes the 

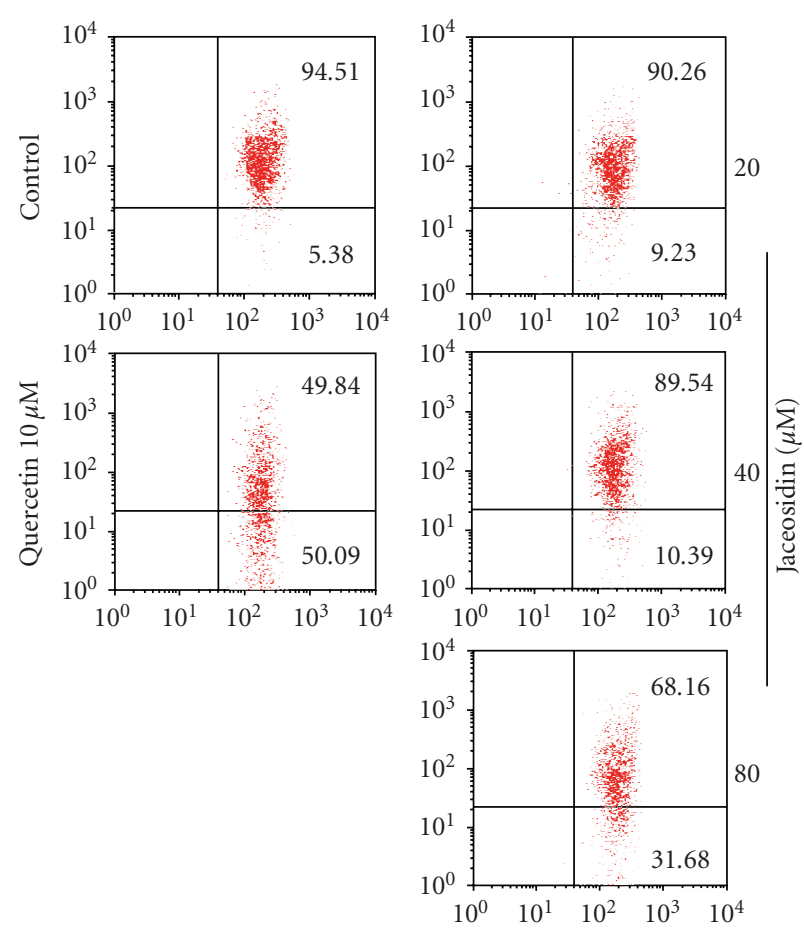

(a)
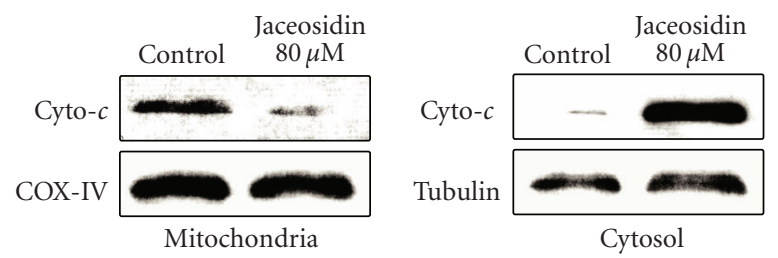

(b)

FIgURE 3: Jaceosidin dose-dependently (a) induces loss of mitochondrial membrane potential and (b) induces cytochrome $c$ to release from mitochondria to cytosol. CAOV-3 cells were seeded into 12-well-plate and cultured overnight. Jaceosidin or quercetin was added into each well. Twenty-four hours later, cells were harvested for measuring mitochondrial membrane potential by flow cytometry using JC-1 staining. Cells on the lower side showed lower membrane potential. One of three independent experiments is shown. In addition, mitochondria and cytosol proteins were prepared from cells treated with $80 \mu \mathrm{M}$ jaceosidin, which were subjected to western blotting for measuring cytochrome $c$ in mitochondria and cytosol.

mitochondrial pathway. As expected, the next experiment further demonstrated that jaceosidin treatment greatly induced the cytochrome $c$ to release from mitochondria to cytosol in the cells (see Figure 3(b)), indicating involvement of the intrinsic apoptosis route via mitochondria.

Once released into the cytoplasm, cytochrome $c$ usually interacts with Apaf-1 (apoptotic protease-activating factor1), adenosine triphosphate-deoxyadenosine triphosphate (ATP/dATP), and procaspase-9 to form a complex known as the apoptosome [24]. In the apoptosome, caspase-9 is activated which in turn activates effector caspases, like procaspase-3 and -7 [25]. The effector caspases may cleave

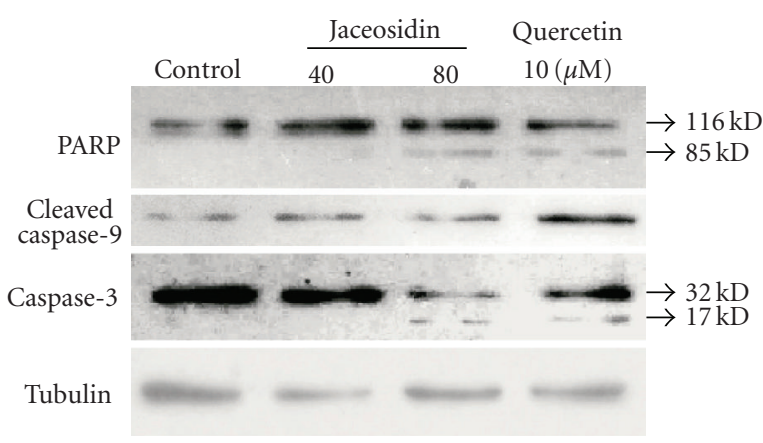

FIGURE 4: Jaceosidin activates caspase-3 and its related proteins to promote apoptosis. Whole cell lysates prepared from CAOV-3 cells, which had been incubated with jaceosidin or quercetin for 24 hours. The proteins were subjected to Western blot analysis using the respective antibodies as indicated. Data are representative of three separate experiments.

a number of structural and regulatory cellular proteins including PARP and lamin protein and are responsible for the typical morphologic and biochemical features of an apoptotic cell [26, 27]. Figure 4 showed the effects of jaceosidin on these events occurred in the apoptosis, where jaceosidin markedly caused increases in the cleaved form of PARP, caspase-3, and caspase-9. This result further confirmed that jaceosidin promotes apoptosis through capase-3 via mitochondria. Walle et al. reported that 5, 7dimethoxyflavone and 5, 7, 4' -trimethoxyflavone were both 10 times more potent inhibitors of cell proliferation than the corresponding unmethylated analogs chrysin and apigenin, fully methylated flavones appear to have great potential as cancer chemopreventive/chemotherapeutic agents [28]. However, in our experiments, quercetin $\left(3,3^{\prime}, 4^{\prime}, 5\right.$, 7-pentahydroxyflavone) showed obviously better efficacy than jaceosidin (4', 5, 7-trihydroxy-3', 6-dimethoxyflavone). Comparison of the structure of these compounds suggested that 3-hydroxyl group might be very important for the function of flavone compounds. Further experiments need to be done to confirm this hypothesis.

Overall, our study has proved an antitumour effect of jaceosidin, a flavone from Artemisia vestita Wall. Its mechanism underlying the effect mainly involves the induction of apoptosis through activating caspase- 3 via mitochondrial pathway. The findings obtained here may be helpful for understanding the properties of jaceosidin as a candidate of antitumour drug.

\section{ACKNOWLEDGMENT}

This study was supported by National Natural Science Foundation of China (no. 30672656 and 30672472).

\section{REFERENCES}

[1] I. B. Weinstein, "Disorders in cell circuitry during multistage carcinogenesis: the role of homeostasis," Carcinogenesis, vol. 21, no. 5, pp. 857-864, 2000. 
[2] T. Jacks and R. A. Weinberg, "Taking the study of cancer cell survival to a new dimension," Cell, vol. 111, no. 7, pp. 923-925, 2002.

[3] C. Chen and A.-N. T. Kong, "Dietary cancer-chemopreventive compounds: from signaling and gene expression to pharmacological effects," Trends in Pharmacological Sciences, vol. 26, no. 6, pp. 318-326, 2005.

[4] J. M. Cline and C. L. Hughes Jr., "Phytochemicals for the prevention of breast and endometrial cancer," Cancer Treatment and Research, vol. 94, pp. 107-134, 1998.

[5] M.-S. Kim, H.-J. Kang, and A. Moon, "Inhibition of invasion and induction of apoptosis by curcumin in $\mathrm{H}$-ras-transformed MCF10A human breast epithelial cells," Archives of Pharmacal Research, vol. 24, no. 4, pp. 349-354, 2001.

[6] U. Wenzel, S. Kuntz, M. D. Brendel, and H. Daniel, "Dietary flavone is a potent apoptosis inducer in human colon carcinoma cells," Cancer Research, vol. 60, no. 14, pp. 3823-3831, 2000.

[7] T. Nakasugi, M. Nakashima, and K. Komai, "Antimutagens in gaiyou (Artemisia argyi Levl. et Vant.)," Journal of Agricultural and Food Chemistry, vol. 48, no. 8, pp. 3256-3266, 2000.

[8] G. R. Schinella, R.-M. Giner, M. Del Carmen Recio, P. M. De Buschiazzo, J.-L. Ríos, and S. Máñez, "Anti-inflammatory effects of South American Tanacetum vulgare," Journal of Pharmacy and Pharmacology, vol. 50, no. 9, pp. 1069-1074, 1998.

[9] H.-J. Seo, K.-K. Park, S. S. Han, et al., "Inhibitory effects of the standardized extract (DA-9601) of Artemisia asiatica Nakai on phorbol ester-induced ornithine decarboxylase activity, papilloma formation, cyclooxygenase- 2 expression, inducible nitric oxide synthase expression and nuclear transcription factor $\kappa \mathrm{B}$ activation in mouse skin," International Journal of Cancer, vol. 100, no. 4, pp. 456-462, 2002.

[10] Y. Sun, Y. H. Li, X. X. Wu, et al., "Ethanol extract from Artemisia vestita, a traditional Tibetan medicine, exerts anti-sepsis action through down-regulating the MAPK and NF- $\kappa \mathrm{B}$ pathways," International Journal of Molecular Medicine, vol. 17, no. 5, pp. 957-962, 2006.

[11] M. A. Jeong, K. W. Lee, D.-Y. Yoon, and H. J. Lee, “Jaceosidin, a pharmacologically active flavone derived from Artemisia argyi, inhibits phorbol-ester-induced upregulation of COX-2 and MMP-9 by blocking phosphorylation of ERK-1 and -2 in cultured human mammary epithelial cells," Annals of the New York Academy of Sciences, vol. 1095, no. 1, pp. 458-466, 2007.

[12] H.-G. Lee, K.-A. Yu, W.-K. Oh, et al., "Inhibitory effect of jaceosidin isolated from Artemisia argyi on the function of E6 and E7 oncoproteins of HPV 16," Journal of Ethnopharmacology, vol. 98, no. 3, pp. 339-343, 2005.

[13] M.-J. Kim, D.-H. Kim, K. W. Lee, D.-Y. Yoon, and Y.-J. Surh, "Jaceosidin induces apoptosis in ras-transformed human breast epithelial cells through generation of reactive oxygen species," Annals of the New York Academy of Sciences, vol. 1095, no. 1, pp. 483-495, 2007.

[14] R. N. Buick, R. Pullano, and J. M. Trent, "Comparative properties of five human ovarian adenocarcinoma cell lines," Cancer Research, vol. 45, no. 8, pp. 3668-3676, 1985.

[15] H. Morimoto, J. T. Safrit, and B. Bonavida, "Synergistic effect of tumor necrosis factor- $\alpha$ - and diphtheria toxin-mediated cytotoxicity in sensitive and resistant human ovarian tumor cell lines," The Journal of Immunology, vol. 147, no. 8, pp. 2609-2616, 1991.

[16] M. E. Kaighn, K. S. Narayan, Y. Ohnuki, J. F. Lechner, and L. W. Jones, "Establishment and characterization of a human prostatic carcinoma cell line (PC-3)," Investigative Urology, vol. 17, no. 1, pp. 16-23, 1979.
[17] W. F. Scherer, J. T. Syverton, and G. O. Gey, "Studies on the propagation in vitro of poliomyelitis viruses. IV. Viral multiplication in a stable strain of human malignant epithelial cells (strain HeLa) derived from an epidermoid carcinoma of the cervix," The Journal of Experimental Medicine, vol. 97, pp. 695-710, 1953.

[18] J. F. Kerr, A. H. Wyllie, and A. R. Currie, "Apoptosis: a basic biological phenomenon with wide-ranging implications in tissue kinetics," British Journal of Cancer, vol. 26, no. 4, pp. 239-257, 1972.

[19] Y. A. Hannun, "Apoptosis and the dilemma of cancer chemotherapy," Blood, vol. 89, no. 6, pp. 1845-1853, 1997.

[20] S. Sen and M. D’Incalci, "Apoptosis biochemical events and relevance to cancer chemotherapy," FEBS Letters, vol. 307, no. 1, pp. 122-127, 1992.

[21] D. R. Green and J. C. Reed, "Mitochondria and apoptosis," Science, vol. 281, no. 5381, pp. 1309-1312, 1998.

[22] M. Castedo, A. Macho, N. Zamzami, et al., "Mitochondrial perturbations define lymphocytes undergoing apoptotic depletion in vivo," European Journal of Immunology, vol. 25, no. 12, pp. 3277-3284, 1995.

[23] A. B. Granado-Serrano, M. A. Martín, L. Bravo, L. Goya, and S. Ramos, "Quercetin induces apoptosis via caspase activation, regulation of Bcl-2, and inhibition of PI-3-kinase/Akt and ERK pathways in a human hepatoma cell line (HepG2)," The Journal of Nutrition, vol. 136, no. 11, pp. 2715-2721, 2006.

[24] P. Li, D. Nijhawan, I. Budihardjo, et al., "Cytochrome c and dATP-dependent formation of Apaf-1/caspase-9 complex initiates an apoptotic protease cascade," Cell, vol. 91, no. 4, pp. 479-489, 1997.

[25] S. M. Srinivasula, M. Ahmad, T. Fernandes-Alnemri, and E. S. Alnemri, "Autoactivation of procaspase- 9 by Apaf-1-mediated oligomerization," Molecular Cell, vol. 1, no. 7, pp. 949-957, 1998.

[26] W. C. Earnshaw, L. M. Martins, and S. H. Kaufmann, "Mammalian caspases: structure, activation, substrates, and functions during apoptosis," Annual Review of Biochemistry, vol. 68, pp. 383-424, 1999.

[27] D. W. Nicholson, "Caspase structure, proteolytic substrates, and function during apoptotic cell death," Cell Death \& Differentiation, vol. 6, no. 11, pp. 1028-1042, 1999.

[28] T. Walle, N. Ta, T. Kawamori, X. Wen, P. A. Tsuji, and U. K. Walle, "Cancer chemopreventive properties of orally bioavailable flavonoids-methylated versus unmethylated flavones," Biochemical Pharmacology, vol. 73, no. 9, pp. 1288-1296, 2007. 

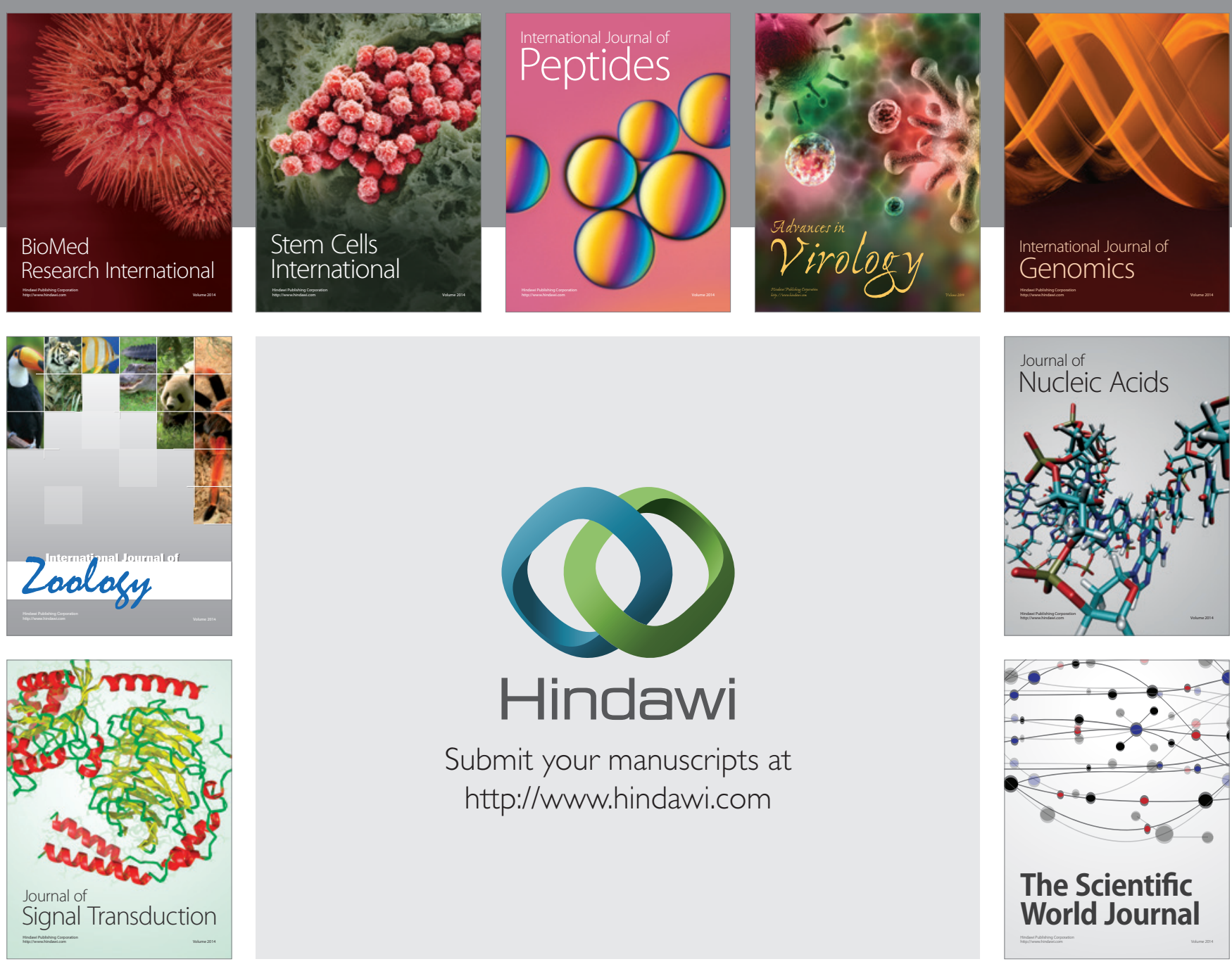

Submit your manuscripts at

http://www.hindawi.com
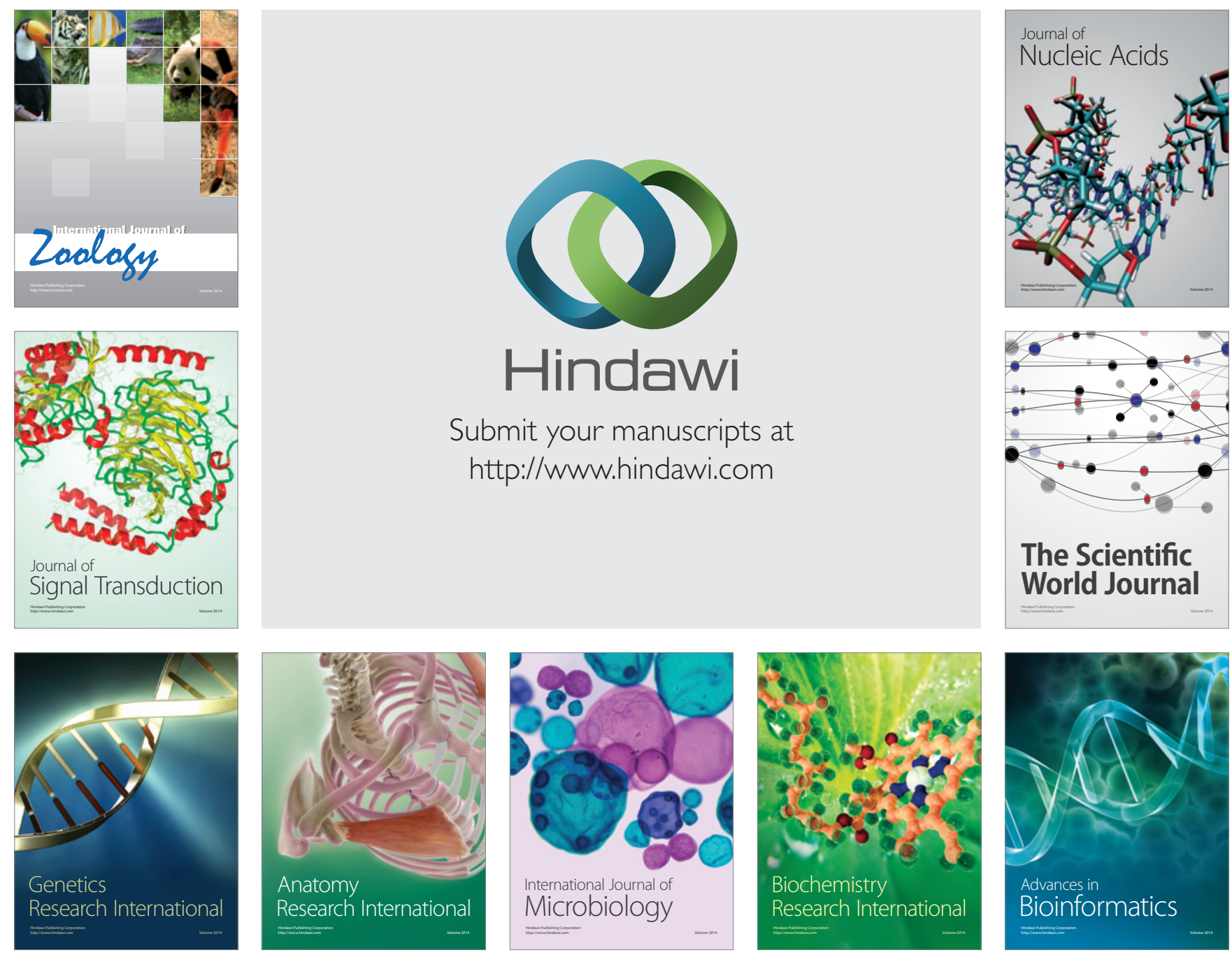

The Scientific World Journal
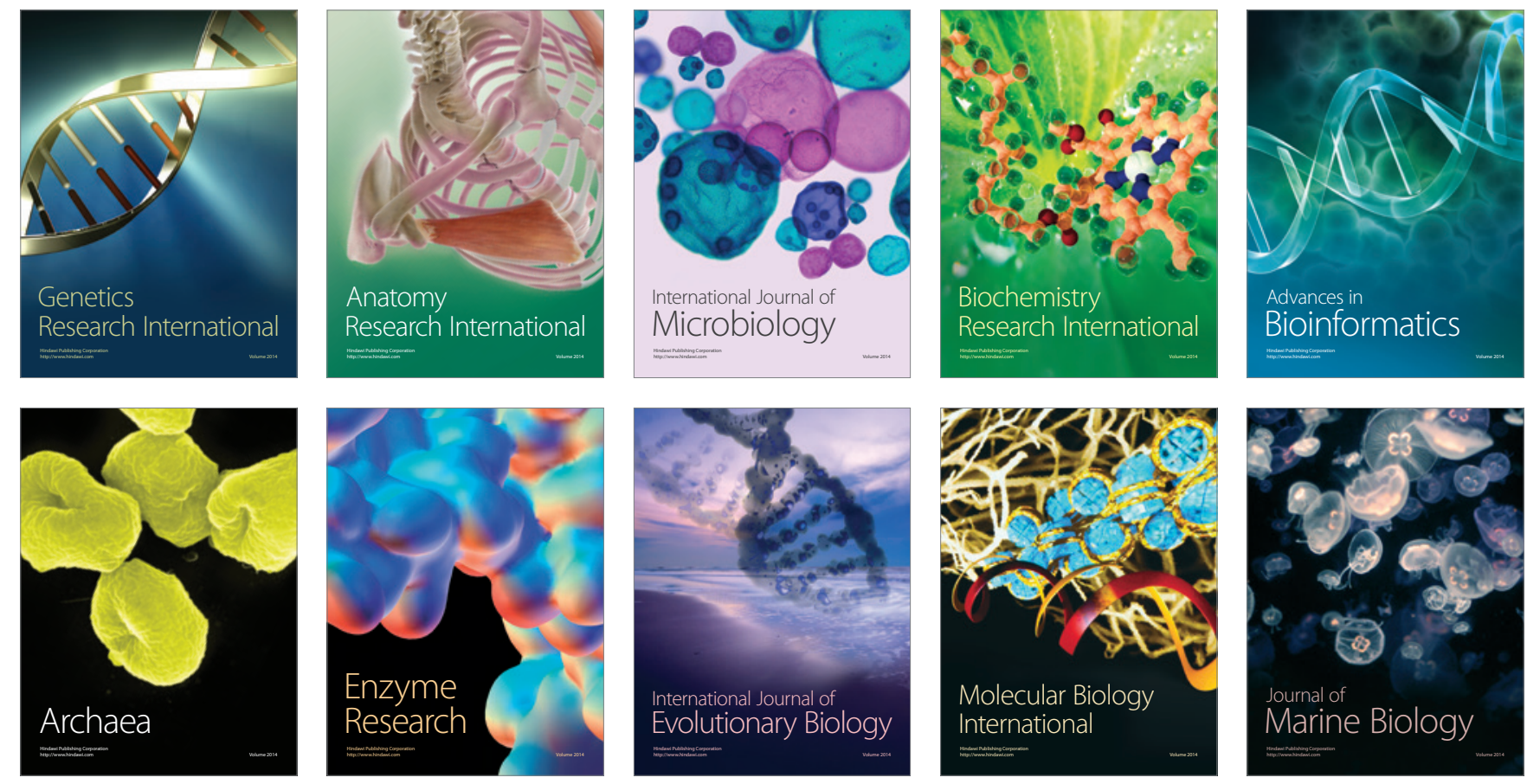